\title{
An Emerging Role of Chatbot in Businesses as a Novel Interactive Tool
}

\author{
T. Venkat Narayana Rao ${ }^{1}$,Vajroju Jyothsna ${ }^{2}$,Sakilam Jhansi Laxmi ${ }^{3}$ \\ Professor $^{1}$, Student ${ }^{2,3}$, Department of C.S.E, SNIST \\ Sreenidhi Institute of Science and Technology \\ Yamnampet,Hyderabad, T.S,India.
}

\begin{abstract}
Chatbot enables the business people to reach their target customers using popular messenger apps like Facebook, Whatsapp etc. Chatbots are not handled by humans directly. Nowadays, Chatbots are becoming very popular especially in business sector by reducing the human efforts and automated customer service. It is a software which interacts with user using natural language processing, Machine Language and Artificial Intelligence. They allow users to simply ask questions which would simulate interaction with the humans. The popular and well known chatbots are Alex and Siri. This paper focus on review of chatbot, history of chatbot and its implementation along with applications.
\end{abstract}

$* * * * *$

\section{Introduction}

A chatbot is an artificial person or other creature which communicates with users. It may be a text-based conversation or a spoken conversation. It can run on local computers and phones, though most of the time it is retrieved through the internet. It is also exhibited as one of the most advanced interactions between humans and machines. It is interesting and inspiring to develop and work with the chatbot.

As the industry grows, more competitors will enter into the market and growth will be exponential[1]. Chatbots can be used for different purposes like it may be used as a personal stylist that recommends the user what suits on their body based on skin colour and other measurements. Chatbots are used in ordering food and helps business people in reminding their meetings their appointments, emails and maintaining business dairies. Now in this paper, we would discuss the uses of the chatbot in the business field.

We, the humans, prefer to acquire information right at our fingertips. A chatbot is a tool which can reduce work of both the users and the business persons. A chatbot is a manual program outlined to replicate a conversation through text or speech[1][2]. It takes text or speech as an input and delivers output either via text, speech, images. When the user types "Do you accept cash on delivery for your products ? " bot replies something similar to this "Yes, we accept COD only for the products which cost above Rs.499". Based on the queries raised by the user, it searches for the relevant keywords in the database and displays accurate information which is already stored.

The history of chatbot:

- 1950-The Turing Test: Alan Turing conjectured that a machine would equally exhibit intelligent behaviour as that of the human. This idea essentially laid the foundation for chatbots.

- 1966-ELIZA was the initial chatbot, created in 1966 by Joseph Weizenbaum. The role of it was to be a
Therapist. The purpose of this bot was to simulate human conversation by matching user prompts and scripted responses. Despite the fact that it lacked "real" intelligence, it gave an illusion of understanding about chatbots.

- 1972-PARRY was designed by American psychiatrist Kenneth Colby in 1972. While ELIZA simulated a therapist, PARRY simulated a patient with schizophrenia. It attempts to simulate the disease. It is a natural language program that resembles the thinking of an individual.

- 1988-Jabberwacky attempted at simulating a human conversation through Artificial Intelligence. It was purely for an entertainment purpose.

- 1992-Dr.Sbaitso was a chatbot created for MS-DOS. It was purely operated by the voice which simulated the liveliness of a Doctor around. It played the role of Psychologist.

- 1995-ALICE "Artificial Linguistic Internet Computer Entity" which was generally coined ALICE. Although it was unable to pass the Turing test, it was able to impress users through natural Language processing and heuristic pattern matching.

- 2001-SMARTERCHILD was a precursor to Siri. It was able to handle out fun conversations with easy and faster data access to other services.

Later IBM WATSON, SIRI, GOOGLE, ALEXA, CORTANA, TAY are the recent chatbots implemented by observing the necessity and usage in our present day-to-day life.

\section{Architecture}

Imagine for a minute what the process for communication with another human being is like.

Assume your boss asks you to buy some 100\% Apple Juice. 
1. Your first question is how much of it does he/she want?

2. 1 liters? 500ml? 200? And finally - mentions 1 liter of 100\% Apple Juice.

3. You find one store, and go back to your boss and tell him/her you found what is needed.

4. It's Rs.120 and do the finish the purchase.

A Chabot follows the same process, with two fundamental differences, the channel of communication and what you're talking to. I'll give you a step by step on the most fundamental principles of $\mathrm{AI} / \mathrm{Ch}$ atbots based on the image below i.e. figure 1.

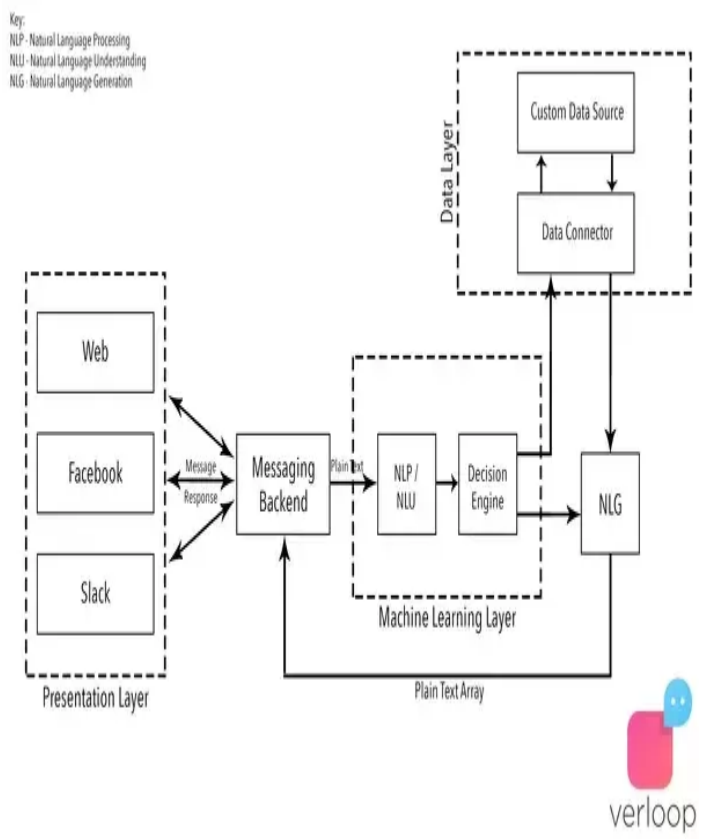

Figure 1: Architecture of Chatbot

1. You find a product on Facebook's Messenger, for the sake of consistency; let's assume it's the same bottle of Juice. You can only see the presentation layer and send a message to the bot.

2. Using Natural Language Processing, the machine transforms basic text request into structured commands.

3. Now the data is dumped into a decision engine so as to meet certain conditions to exit conversational loop, particularly, the quantity of juice you want.

4. Using Natural Language Generation (what happens when computers write a language. NLG processes structured data into text), similar to what you did with your boss, and the bot asks you how much of juice is required.

5. This list of reply reaches back into the messaging backend (as shown in Fig.1) and is shown to you as a question form. You tell the bot you want 1 liter and get back to the decision engine through NLP [3][7].

6. The bot analyzes data that is fed before about the product, stores, their locations and their proximity to your location. It identifies the nearest store that has this product available and tells you what it costs.
7. It then directs you to a payment page and after it receives confirmation, it places your order for you and delivered in one to two business days, you have 1 liter of Tropicana 100\% Apple Juice.

\section{Chatbot Implementation:}

Chatbot can be implemented by using coding in $\mathrm{C}, \mathrm{C}++$, Java, Python etc. We can create basic chatbot for our business in less time by using the resources listed below. The below listed resources require no coding[8][9].

Chatfuel: We can build chatbot using Chatfuel in just a few minutes. It does not require any coding. It specializes in developing chatbots for Facebook Messenger. It is simple and easy to use. But it has some disadvantages. It does not validate parameters like mobile number, email etc.

Botsify: Botsify allows us to use build chatbot especially for Facebook messenger in few minutes. It can validate parameters such as phone number and email. It is better than Chatfuel. It is free to use for unlimited messages, pricing plans start when we want to integrate with other platforms [3].

Conversable: Conversable is a conversational platform which allows one to create intelligent and automated responses in social platforms.

Drift: Drift is the world's first and only conversational marketing platform. It helps us to connect with and qualify our leads in real time.

There are some other resources which help in creating Chatbot. Some of them are Gupshup, Manychat and MobileMonkey etc.

\section{Chatbot Development Platforms}

Chatbot can be developed by using some platforms. It requires some coding knowledge. These platforms consist of tools like Natural Language Processing, Artificial intelligence, Machine learning at the ready. The below listed are some of the Chatbot Development Platforms:

Facebook Messenger Platform: Instead of opening an app, making a phone call, searching on the Internet, and running for service, customer can just ask a query in Facebook Messenger. It can reach many people. It save time and money for hassle free customer service [4].

Dialogflow: Dialogflow is also known as API. AI, which is owned by Google. It allows customers to interact with our 
product by building engaging voice and text-based conversational interface powered by Artificial Intelligence.

IBM Watson: Watson allows us to integrate the most powerful AI into our application. It allows us to apply advanced Machine Learning, Artificial Intelligence, Natural Language Processing and Information Retrieval [9].

Amazon Lex: Amazon Lex allows us to build an application that can engage customers and can interact in a lively manner. It provides to integrate with AWS Lamda, AWS MobileHub etc.

Some other chatbot development platforms are LUIS, Pandorabots, Chatbase and Azure Bot framework.

\section{Proposed Algorithm (Using facebook messenger and} API.AI):

1. Start

2. Generate a page access token in Facebook developer page.

3.Link the Facebook page and API.AI.

4. Create new Intents, Entities required to develop Bot

5. Add various queries and answers to different questions which user will be asking.

6. Train the bot by adding various kinds of questions and answers which in turn improves the efficiency of the bot.

7. Try adding different Testers in training a bot because an individual alone may identify different queries related to our application.

8. Stop.

\section{Merits}

Chatbots have obtained more popularity in the marketing industry and in this way they have provided a voguish way for business to converse with the world and especially with their customers, wiping out the popularity of messaging apps. Adopting the chatbot technology will give us a major advantage as a marketer over competitors [5].

Some of the benefits of using chatbot are:-

A. Improved customer service: A survey shows $85 \%$ of online shopping customers need help in their shopping. So buyers may need assistance in attempting to understand which commodity meets their needs. It will provide a real-time assistant for the customers like a salesperson in the stores and also offer interactive communication where they can also ask queries to acknowledge the real issue. Customers can convey their messages using text or voice.

B. Better Engagement: It is important for the companies to engage customers with their brands by using social media marketing and by this, the sales of all the companies will increase by $20 \%$ to $30 \%$. So, chatbot enables a better experience and helps the customers to maintain better engagement.

C.Cost Savings: Rather than creating a cross-platform app or recruiting workers for every quest, implementing the chatbot functionality is quicker and inexpensive. Whereas chatbot allows corporations to tackle many buyers at once and concurrently. By using chatbot, companies will not only save the employee cost but also they can avoid the problems caused by human errors.

D. Rapid and increasing growth in Messenger app: Half of the smartphone users do not download the new apps. Since the core of people are using WhatsApp, Facebook etc. apps more frequent, instead of creating a new app they can integrate chatbot in one of the most popular apps which can save time and money.

As there are some advantages there may be some disadvantages using chatbot in the business field.

\section{Demerits}

A. Limited responses for customers: The customers may not get always the answers they are searching for because chatbot is a template in which only some questions were programmed while using it.

B. Complex chatbot may cost more: Solving some problems while building complex chatbot may cost higher .

C. Not all business can use Chatbots: Trying to program all the questions or possible scenarios is time taking, costly and in some business, chatbot is not used because they need person to person interactions. Hence, it is true that using chatbot will save money and decreases the worries of the employees but before including it, companies should consider all pros and cons [6].

\section{Conclusion}

Nowadays, customers expect the information they are looking for on internet quickly and easily. When a business cannot provide such kind of experience, customers get frustrated. Chatbots are used to decrease these frustrations by providing a real-time approach that customers are searching. During the technological advancements in AI, buyers are provided with technical help in all facets of their lives. The internet presents diverse ways of getting information and also altered the way of communication. Innovation strengthened our lives with more possibilities. Everyone expects to get quick answers with less hinder. A chatbot is a program or a service that can easily bind with the customer to help in solving some questions. The services 
offered by chatbot can provide information about health and diseases, weather forecasting and helps in purchasing cosmetics, dresses, shoes etc. Interaction with chatbot feign the interaction with a real person.

Chatbots learn to do new things by minimizing the information from the huge data. Chatbot seems to be clever but they are not. They are flexible and predictive in their learning curve. That means if the input is bad or repeats questionable statements, their behaviour will develop accordingly. Chatbots need training and users need to be careful so that they understand the constraints of software they are interacting with. Some of the predictive future of chatbots are development of AI impacts on chatbot, Voice Experiences going mainstream and Social Messenger Applications that would drive Chatbot Marketing.

\section{References:}

[1]. B. Setiaji, F. W. Wibowo, "Chatbot Using A Knowledge in Database", IEEE 7th International Conference on IntelligentSystems, Modelling and Simulation, Thailand, pp. 72-77, 2016.

[2]. Weintraub J., 1986, History of the PC Therapist.

[3]. B. A. Shawar and E. Atwell, "Chatbots: are they really useful?", LDV

[4]. Abu Shawar, Bayan and Eric Atwell. 2004b. A chatbot as a novel corpus visual- ization tool. In M. Teresa Lino, M. Francisca Xavier, F. Ferreira, R. Costa, R. Silva, C. Pereira, F. Caevalho, M. Lopes, M. Catarino and S. Barros (eds.). Proceedings of Fourth International Conference on Language Resources and Evaluation (LREC'04) IV, 2057-2060. Paris: ELRA.

[5]. Gimeno A(2008).The EUROCALL Review.An Evaluation of Chatbots as Aids to Learning English as a second Language .Downloaded on 20/2/2011. from:http://www.eurocall-languages.org/review/index.html.

[6]. Surmenok, P.: Chatbot Architecture, March 10, 2017.

[7]. Jia, J. (2003). The Study of the Application of a Keywords-based Chatbot System on the Teaching of Foreign Languages. ArXiv preprint cs/0310018.

[8]. Sansonnet, J.-P., Leray, $\quad$ D., $\quad \& \quad$ Martin, J.-C. (2006). Architecture of a Framework for Generic Assisting Conversational Agents. Intelligent Virtual Agents Lecture Notes in Computer Science, 145-156

[9]. Anirudh Khanna, Bishwajeet Pandey ,"A Study of Today's A.I. through Chatbots and Rediscovery of Machine Intelligence," International Journal of u-ande-Service, Science and Technology Vol.8, No. 7 (2015), pp.277-284. 